\title{
Three-dimensional volumetric gray-scale uterine cervix histogram prediction of days to delivery in full term pregnancy
}

\author{
Ji Youn Kim, Hai-Joong Kim, Meong Hi Hahn, Hye Jin Jeon, Geum Joon Cho, Sun Chul Hong, Min Jeong Oh \\ Department of Obstetrics and Gynecology, Korea University College of Medicine, Korea
}

\section{Objective}

Our aim was to figure out whether volumetric gray-scale histogram difference between anterior and posterior cervix can indicate the extent of cervical consistency.

\section{Methods}

We collected data of 95 patients who were appropriate for vaginal delivery with 36th to 37th weeks of gestational age from September 2010 to October 2011 in the Department of Obstetrics and Gynecology, Korea University Ansan Hospital. Patients were excluded who had one of the followings: Cesarean section, labor induction, premature rupture of membrane. Thirty-four patients were finally enrolled. The patients underwent evaluation of the cervix through Bishop score, cervical length, cervical volume, three-dimensional (3D) cervical volumetric gray-scale histogram. The interval days from the cervix evaluation to the delivery day were counted. We compared to 3D cervical volumetric gray-scale histogram, Bishop score, cervical length, cervical volume with interval days from the evaluation of the cervix to the delivery.

\section{Results}

Gray-scale histogram difference between anterior and posterior cervix was significantly correlated to days to delivery. Its correlation coefficient $(R)$ was $0.500(P=0.003)$. The cervical length was significantly related to the days to delivery. The correlation coefficient $(R)$ and $P$-value between them were 0.421 and 0.013 . However, anterior lip histogram, posterior lip histogram, total cervical volume, Bishop score were not associated with days to delivery $(P>0.05)$.

\section{Conclusion}

By using gray-scale histogram difference between anterior and posterior cervix and cervical length correlated with the days to delivery. These methods can be utilized to better help predict a cervical consistency.

Keywords: Prediction of delivery day; Three-dimensional ultrasoundgraphy; Three-dimensional volumetric gray-scale histogram

\section{Introduction}

Prediction of labor duration is important for the evaluation and management of pregnancy, and the condition of the cervix, which can be evaluated with various techniques, is crucial for predicting the duration of labor.

The most common evaluation method is the Bishop score, which is determined by manual pelvic examination. The Bishop score is used to decide whether or not to induce labor, and the total score is composed of five components which include cervical dilation, effacement, consistency,
Received: 2013.2.25. Revised: 2013.5.16. Accepted: 2013.6.7.

Corresponding author: Hai-Joong Kim

Department of Obstetrics and Gynecology, Korea University Ansan Hospital, Korea University College of Medicine, 123 Jeokgeum-ro, Danwon-gu, Ansan 425-707, Korea

Tel: +82-31-412-5531 Fax: +82-31-412-4233

E-mail: haijkim@gmail.com

Articles published in Obstet Gynecol Sci are open-access, distributed under the terms of the Creative Commons Attribution Non-Commercial License (http://creativecommons. org/licenses/by-nc/3.0/) which permits unrestricted non-commercial use, distribution, and reproduction in any medium, provided the original work is properly cited.

Copyright (C) 2013 Korean Society of Obstetrics and Gynecology 


\section{Obstetrics \& Gynecology Science}

Ji Youn Kim, et al. 3D volumetric uterine cervix histogram prediction of delivery

position and fetal station. Although this method is the most cost-effective and commonly used, controversy about its accuracy persists [1-6].

Another method of evaluation used to predict preterm delivery is ultrasonographic measurement of the length of the cervix. However, despite its frequent use, many studies have indicated that there is a wide range of cut off values for this procedure, which are less significant for low risk groups [79]. Cervical funneling, another widely used ultrasonographic marker, has also had controversial results $[10,11]$. Both cervical length measurements and the funneling method lack information about cervical ripening and have been shown to be insufficient for predicting labor.

Recently, several studies have claimed that analysis via cervical gray-scale histogram and cervical volume measurement are useful tools for predicting delivery. Although the cervical volume study results remain controversial [12-17], most analysis of the cervical gray-scale histogram has indicated that it is an effective tool [18-22].

A gray-scale histogram of the cervix measures cervical density based on the tissue echogenicity, which indicates cervical consistency. The gray-scale histogram measurements depend on the position and size of the measurement points when performed at a two-dimensional (2D) level. This study analyzed three-dimensional (3D) volumetric gray-scale histograms of the entire cervix and used the differences between the anterior and posterior cervix to increase accuracy. The analysis indicated that the anterior cervix was much more echogenic than the posterior cervix. This is because smaller amounts of the ultrasound beam can pass through the anterior lip of the hard cervix. Thus, there is a greater difference between the anterior and posterior cervical gray-scale histograms than between those of the soft cervix [19]. This study evaluated the volumetric gray-scale histogram difference between the anterior and posterior cervix in order to determine the extent and consistency of the cervix.

The objective of this study was to evaluate the relationship between the number of days to delivery and the volumetric gray-scale histogram value. The study evaluated the ability of the difference between the anterior and posterior cervix to predict cervical consistency and compared these values with current methods that include cervical length, volume and Bishop score.

\section{Materials and methods}

This was a prospective study that collected data of 439 patients from September 2010 to October 2011. A total of 34 patients from the Department of Obstetrics and Gynecology at Korea University Ansan Hospital were enrolled in this study, and each patient was 36 to 37 weeks gestational age. Patients that had experienced a Cesarean section, induction of labor or a premature membrane rupture were excluded from this study. Informed consent was obtained from each of the patients, and this study was approved by the Institutional Review Board of the hospital.

The patients underwent cervical evaluation using the Bishop score, cervical length, cervical volume, and a 3D cervical volumetric gray-scale histogram during the 36th to 37th weeks of gestation. Patients were given routine check-ups until delivery, and the study recorded the number of days between the cervical evaluation to the delivery date. The Bishop score was estimated by a single examiner that performed a digital examination. The patients were evaluated by transvaginal ultrasonography in the dorsal lithotomy position with an empty urinary bladder after the pelvic exam. All ultrasound examinations were performed by one experienced sonographer. The transvaginal probe was slowly inserted into the vagina beneath the middle of the cervix, where the cervix was clearly visible, and to avoid distortion of the cervix, no pressure was applied. A sagittal plane of the cervix was identified where the internal os, cervical canal, and the external os were visible. The image was then magnified until the cervix occupied $75 \%$ of the monitor screen [23].

The length of the cervix was measured from the external to the internal cervical os, which showed the entire length of the cervix. Measurements were repeated a total of three times, and the mean of the three values was calculated and used for statistical analysis. The study used an ACCUVIX V20 ultrasound system (Samsung Medison, Seoul, Korea), and this device was also used for the 3D analysis.

The cervix was centralized in the 3D sector that appeared on the ultrasound screen, and the data were obtained while the transducer was stationary and the crystals were mechanically rotated across the sector with a sweep angle of $90^{\circ}$. The fast volume acquisition setting (low resolution) was used to minimize periodic flashing artifacts that could arise from uterine artery pulsation or from fetal movement. The volume was acquired for 15 to 20 seconds, depending 


\title{
Obstetrics \& Gynecology Science
}

\author{
Vol. 56, No. 5, 2013
}
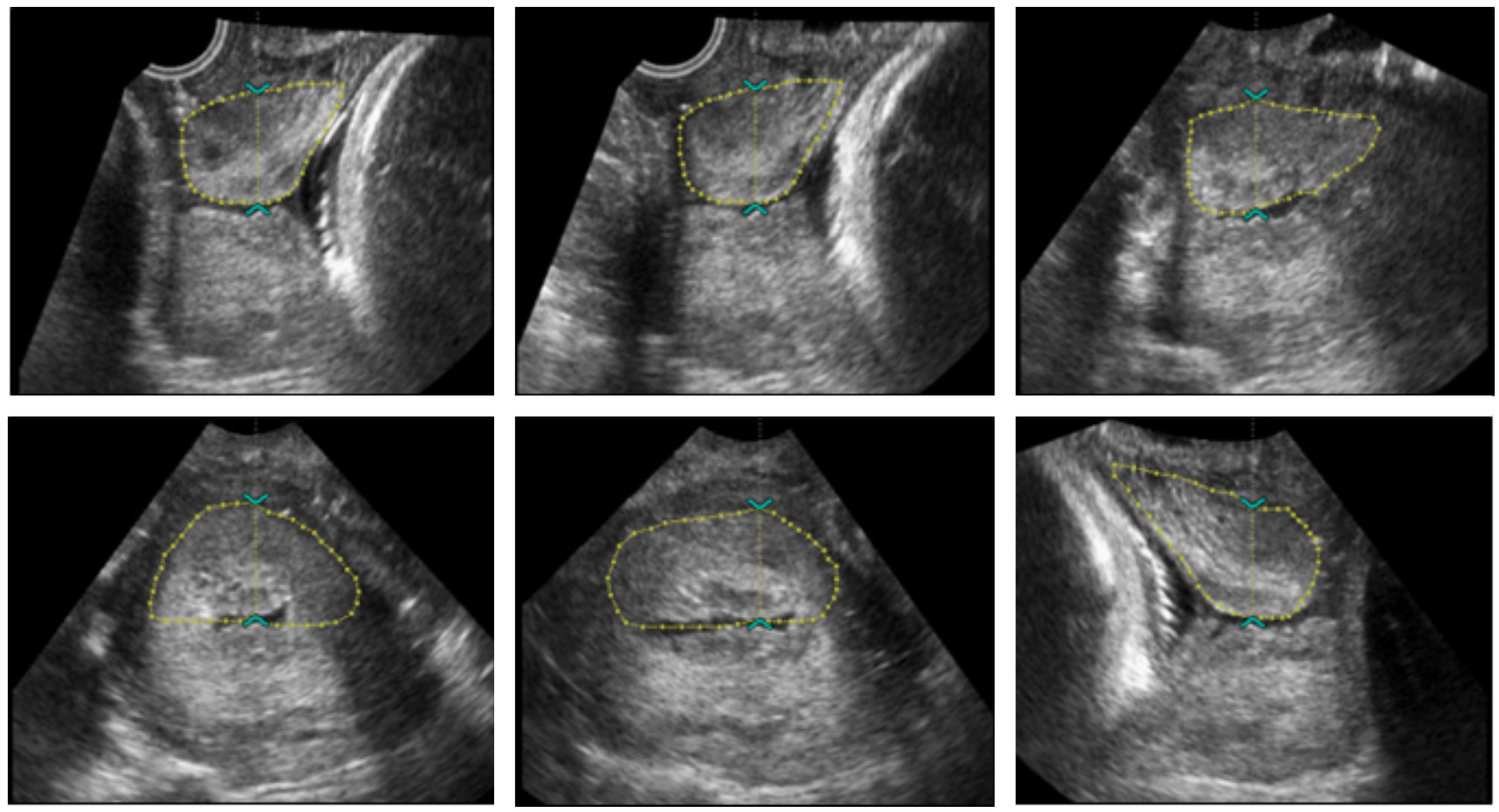

Fig. 1. Six contours of the cervix in $30^{\circ}$ increments of rotation with a roller ball cursor. Six anterior cervix contours in $30^{\circ}$ rotation increments.
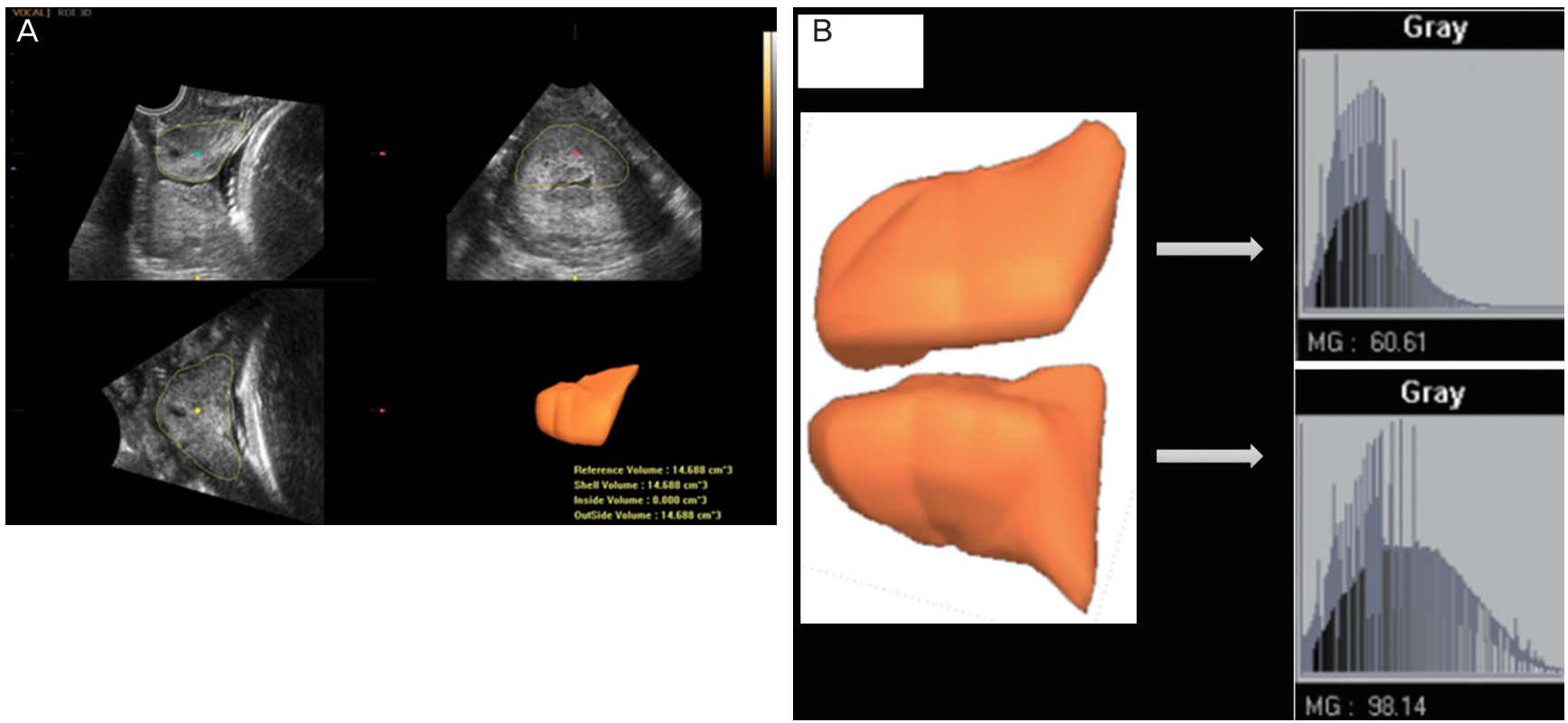

Fig. 2. Three-dimensional ultrasound cervical volume measurement. (A) Multiplanar display of the anterior cervical lip: longitudinal plane in the upper left quadrant, transverse plane in the upper right quadrant and coronal plane in the lower left quadrant. The resultant threedimensional model of the anterior cervix can be seen in the lower right image. (B) Gray-level histogram of each of the lips of the cervix shown at higher magnification. The mean gray-scale histogram was calculated as 32.0, which roughly agreed with the peak gray-level on the histogram. The mean gray-scale histogram represents the echogenicity of the region of interest.

on the dimension of the 3D sector. Cervical volume $\left(\mathrm{cm}^{3}\right)$ was calculated using the Virtual Organ Computer-aided
AnaLysis (VOCAL, Samsung Medison), and the anterior lip and posterior lip volumes were acquired. These volumes 


\section{Obstetrics \& Gynecology Science}

Ji Youn Kim, et al. 3D volumetric uterine cervix histogram prediction of delivery

were manipulated to obtain multi-planar reformatted views of the cervix in the mid-sagittal, axial and coronal planes. All cervical measurements were obtained from the multiplanar images, and the analysis used the VOCAL program manual contour mode. The longitudinal view was used as the reference image, and the rotation steps were performed in increments of $30^{\circ}$ so that six contours of the cervix were manually drawn using the roller ball cursor of the system (Fig. 1). The lower uterine segment, the vaginal wall, and especially the large uterine arteries were not included when the contours were drawn; the study used the hyperechogenic or hypoechogenic line between the cervix and the vaginal wall and the internal and external cervical os as landmarks. Once the contours were drawn, the volume and mean grayscale histogram of the cervix were computed automatically. The mean gray-scale histogram was calculated by dividing the integration of pixels in each bar by the total number of pixels in a 3D region of image (ROI) (Fig. 2). The mean grayscale histogram, which represented the echogenicity, was calculated at the cervical area of the ROI. The 3D cervical volumetric gray-scale histogram values for the anterior and posterior lip were obtained using the described protocol, and then the difference between the two values was calculated $[21,24,25]$.

The cervical characteristics of nulliparous and multiparous patients were compared (parity $\geq 1$ ). The study identified a correlation between 3D cervical volumetric gray-scale histogram value and days after the cervix evaluation to the time of delivery. We also compared these results with the Bishop score, cervical length, and the volume of the cervix with the number of days after the evaluation to the date of delivery.

The statistical analysis was conducted with SPSS ver. 17.0 (SPSS Inc., Chicago, IL, USA). The data analysis was performed using a Mann-Whitney $U$ test, and categorical variables were analyzed using the Spearman correlation coefficient. A $P$-value $<0.05$ was considered to be statistically significant.

Table 1. Demographic characteristics and obstetrical outcomes according to parity

\begin{tabular}{|lcc|}
\hline Variables & Parity $=\mathbf{0}(\mathbf{n}=19)$ & Parity $\geq \mathbf{1}(\mathbf{n}=\mathbf{1 5})$ \\
\hline Age $(\mathrm{yr})$ & $31.00(24-37)$ & $32.00(24-37)$ \\
Parity & & $1.00(1-2)$ \\
Gestational age (day) & $261.00(257-267)$ & $261.00(252-264)$ \\
$\quad$ At sonography & $277.00(270-288)$ & $273.00(264-291)$ \\
At delivery & $16.00(6-27)$ & $13.00(0-28)$ \\
Delivery from sonography & $3.22(2.13-3.83)$ & $3.33(2.91-4.08)$ \\
Birth weight $(\mathrm{kg})$ & & \\
\hline
\end{tabular}

Values are presented as median (interquartile range).

Table 2. Cervical findings by three-dimensional ultrasonography, cervical length and Bishop score in parity $=0$ vs. parity $=1$

\begin{tabular}{|c|c|c|c|}
\hline Variables & Parity $=0(n=19)$ & Parity $\geq 1(n=15)$ & $P$-value \\
\hline \multicolumn{4}{|c|}{ 3D volumetric gray-scale histogram } \\
\hline Anterior lip & 72.13 & 92.08 & 0.003 \\
\hline Posterior lip & 62.10 & 90.05 & 0.000 \\
\hline AP difference & 10.02 & 2.02 & 0.124 \\
\hline \multicolumn{4}{|l|}{ 3D volume } \\
\hline Anterior lip & 18.30 & 20.84 & 0.253 \\
\hline Posterior lip & 22.68 & 26.85 & 0.237 \\
\hline Total volume & 41.00 & 47.70 & 0.207 \\
\hline Cervical length & 2.97 & 2.98 & 0.968 \\
\hline Bishop score & 2.74 & 2.73 & 0.995 \\
\hline
\end{tabular}

3D, three-dimensional; AP difference, volumetric gray-scale histogram difference between anterior and posterior cervix. 


\title{
Obstetrics \& Gynecology Science
}

\author{
Vol. 56, No. 5, 2013
}

\section{Results}

The study evaluated 34 patients, 19 of which were nulliparous and 15 which were multiparous (parity $\geq 1$ ). There were no significant differences in age or obstetric outcomes between the two groups. The median gestational age for both groups was 261 days based on ultrasonographic examination of the cervix, the median gestational age at delivery was 277 days and 273 days for nulliparous and multiparous (parity $\geq 1$ ) patients, respectively, and the median number of days from cervical examination by ultrasonography to delivery (days to delivery) was 16 days for the nulliparous and 13 days for the multiparous groups (Table 1).

The study did not observe any statistically significant differences in total cervical volume, cervical length, or Bishop score based on maternal parity. However, there were significant differences between the nulliparous and multiparous patients when the anterior and posterior lips were examined with the 3D volumetric gray-scale histogram. The results did not indicate a significant difference between the anterior and posterior cervix with the 3D volumetric gray-scale histogram (Table 2).

Table 3 shows the results of the bivariate correlation on the association between each cervical factor and the number of days to delivery. The 3D volumetric gray-scale histogram difference between the anterior and posterior cervix was significantly correlated with the number of days to delivery $(R=0.4$,
$P=0.01)$ (Fig. 3). Although the $R^{2}$-value was low, the results indicated that the length of the cervix was significantly related to the time to delivery $(R=0.38, P=0.02)$ (Fig. 3). However, the results did not identify an association between anterior lip histogram, posterior lip histogram, anterior lip volume, posterior lip volume, total cervical volume, or Bishop score with the number of days to delivery (Table 3 ).

Table 3. Bivariate correlation between cervical findings and days to delivery in total study group

\begin{tabular}{|lll|}
\hline \multirow{2}{*}{ Variables } & \multicolumn{2}{c|}{ Days to delivery } \\
\cline { 2 - 3 } & $\boldsymbol{R}$ & $\boldsymbol{P}$-value \\
\hline 3D volumetric gray-scale histogram & & \\
Anterior lip & -0.042 & 0.813 \\
Posterior lip & -0.304 & 0.080 \\
AP difference & 0.500 & 0.003 \\
3D volume & & \\
Anterior lip & 0.159 & 0.369 \\
Posterior lip & -0.013 & 0.940 \\
Total volume & 0.068 & 0.701 \\
Cervical length & 0.421 & 0.013 \\
Bishop score & -0.312 & 0.073 \\
\hline
\end{tabular}

3D, three-dimensional; AP difference, volumetric gray-scale histogram difference between anterior and posterior cervix; $R$, Pearson's correlation coefficiency.
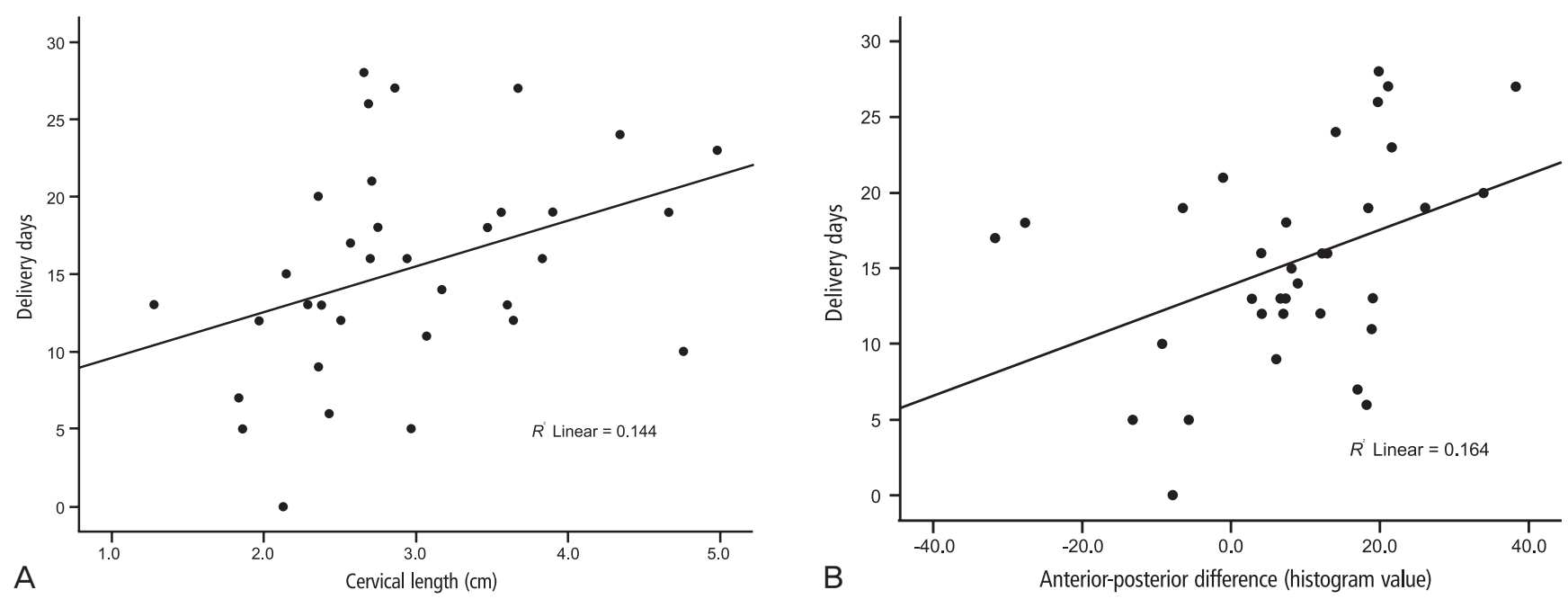

Fig. 3. Correlations between the cervical length and differences of the anterior and posterior cervix with days to delivery using a threedimensional gray-scale histogram. (A) Cervical length and days to delivery $(R=+0.38, P=0.02, Y=2.931[\mathrm{x}]+6.700)$. (B) Anterior-posterior histogram difference and delivery day $(R=+0.40, P=0.01, Y=0.183[\mathrm{x}]+13.890)$. 


\section{Obstetrics \& Gynecology Science}

Ji Youn Kim, et al. 3D volumetric uterine cervix histogram prediction of delivery

\section{Discussion}

Due to the risk factors and physiological mechanisms that are related to preterm delivery, new methods that evaluate the condition of the cervix are important for patient care. Currently, new evaluation methods include 2D or 3D ultrasonography, which focuses on the morphologic change of the cervix using cervical volume [12-17] and a gray-scale histogram [18-22].

Cervical consistency is maintained by increased synthesis of collagen, proteins, glycosaminoglycans (GAGs) and fibronectin within the extracellular matrix of the cervix. Biochemical modifications occur during cervical ripening which affect the arrangement of the cervical tissue. These modifications include a marked reduction of collagen concentration, a 2.5-fold increase in the GAG content, a significant decrease in dermatan sulfate concentrations from $41 \%$ to $15 \%$ of the total GAG content, a 12-fold increase in hyaluronate concentrations, and a marked reduction of fibronectin, all of which have been demonstrated by immunohistochemical experiments. Thus, the loss of collagen and sulfated GAGs may facilitate tension in the ripened cervix, while the significant gain in hyaluronate associated with hydration could explain the soft and swollen consistency of the cervix $[20,26]$.

The graphic representation of the gray-scale histogram illustrates the quantity, reflexivity and disposition of the 'pixels' in the specific area of interest [18]. Although the majority of previous studies measured only the anterior or posterior lips of the cervix or both with a 2D view, a study by Kuwata et al. [19] assessed the differences between the anterior and posterior cervix with a gray-scale histogram. The study determined that a greater positive anterior to posterior value indicated a significantly lower Bishop sub-score for cervical consistency. This result is attributable to the ultrasound beam because, when the beam passes through soft tissue, it indicates low echogenicity. In the soft cervix, the anterior lip allows a greater amount of the ultrasound beam to pass through the tissue, which results in increased echogenicity in the posterior lip. Thus, increased echogenicity of the posterior lip compared with the anterior lip indicates a soft cervix [19].

Previous studies showed that the ultrasound gray-scale histogram can be an objective evaluation method of cervical consistency. However, these studies evaluated the cervix with a 2D level perspective and only evaluated specific parts of the cervix [18,20-22]. Therefore, these measurements may not reflect the entire cervix as $2 \mathrm{D}$ measurements may be inaccurate because the images are dependent on the site of observation and may not identify small changes in the tissue. Based on this information, 3D examination is a more objective test and may be more accurate for predicting the time to labor than $2 \mathrm{D}$ examination. This study measured the entire cervix in 3D volumes with a cervical gray-scale histogram and is the first study that investigated cervical volume measurement using a volumetric gray-scale histogram for cervical consistency.

The results indicate that the anterior lip volumetric grayscale histogram and the posterior lip volumetric gray-scale histogram were not significantly associated with the number of days to delivery. However a bivariate analysis indicated that there was a relevant correlation between the volumetric grayscale histogram difference between the anterior and posterior cervix and the number of days to delivery.

The results demonstrated that greater gray-scale histogram difference between the anterior and posterior cervix was associated with greater number of days to delivery. It was expected that a hard cervix would have a larger gray-scale histogram difference between the anterior and posterior cervix due to the histological changes associated with cervical ripening.

We also evaluated the length of the cervix and the cervical volume to predict the interval of days to delivery. Cervical length is commonly used to predict preterm birth and is significantly associated with preterm delivery [7-9], although numerous studies have indicated varied values for determining delivery. The most commonly used cervical length indicator was less than $25 \mathrm{~mm}$, and the most common preterm birth gestational age was less than 35 weeks [7]. Our study confirmed that the isolated use of the cervical length can also be used as a parameter to predict delivery. Although the $R^{2}$ value was low and the study did not determine specific cutoff values, the analysis indicated that the cervical length was correlated with the number of days to delivery.

The volume of the cervix changes during the progression of labor, and this change can be determined with 3D measurements of the cervical volume. Many studies have hypothesized that predicting delivery may be possible by measuring the 3D volume of the cervix, but the methods of these studies were variable and produced conflicting results. However, these results could be attributable to the variability of enrolled participants and technical problems with sonography. In addition, demarcation between the cervix and the lower uterine segment was difficult and caused technical problems 


\section{Obstetrics \& Gynecology Science}

Vol. 56, No. 5, 2013

with sonography. Therefore, studies with a greater number of patients and which measured the entire volume of the cervix had broader implications for patient applications [12-17]. This study did not determine a correlation between the volume of the cervix and the number of days to delivery.

The Bishop score is currently the most commonly used method to determine the amount of time prior to the induction of labor. This score is especially helpful for women that have indications of labor induction. A previous study demonstrated a correlation between the Bishop score and the time of delivery [2]. However, other studies have indicated that the Bishop score is highly subjective, unreliable, and inaccurate because it only classifies the cervix as favorable or unfavorable. In addition, it is difficult to detect minute changes in the cervix or internal os via the Bishop score, and thus there are limitations to accurately predicting the time of delivery [3-5]. This study demonstrated that the Bishop score was not correlated with the number of days to delivery.

This study had several limitations. First, only a small number of women were investigated. Although the results of the grayscale histogram, which evaluated the difference between the anterior and posterior cervix prior to delivery, were significant, the $R^{2}$-values were very low, and the study did not obtain significant results in regard to the anterior, posterior or total cervical volume. Additional studies that involve more participants are needed to confirm the present findings. In conclusion, the use of the 3D volumetric gray-scale histogram difference between the anterior and posterior cervix can be used to evaluate the condition of the cervix prior to labor. This technique can be utilized to predict cervical consistency, which can indicate the condition of the cervix for labor and delivery.

\section{Conflict of interest}

No potential conflict of interest relevant to this article was reported.

\section{References}

1. Radeka G, Novakov-Mikic A, Ivanovic L. The Bishop score and induction of labor. Med Pregl 2002;55:189-94.

2. Teixeira C, Lunet N, Rodrigues T, Barros H. The Bishop score as a determinant of labour induction success: a systematic review and meta-analysis. Arch Gynecol Obstet 2012;286:739-53.

3. Kolkman DG, Verhoeven CJ, Brinkhorst SJ, van der Post JA, Pajkrt E, Opmeer BC, et al. The bishop score as a predictor of labor induction success: a systematic review. Am J Perinatol 2013;30:625-30.

4. Hendrix NW, Chauhan SP, Morrison JC, Magann EF, Martin JN Jr, Devoe LD. Bishop score: a poor diagnostic test to predict failed induction versus vaginal delivery. South Med J 1998;91:248-52.

5. Zelig CM, Nichols SF, Dolinsky BM, Hecht MW, Napolitano PG. Interaction between maternal obesity and Bishop score in predicting successful induction of labor in term, nulliparous patients. Am J Perinatol 2013;30:75-80.

6. Faltin-Traub EF, Boulvain M, Faltin DL, Extermann P, Irion O. Reliability of the Bishop score before labour induction at term. Eur J Obstet Gynecol Reprod Biol 2004;112:178-81.

7. Crane JM, Hutchens D. Transvaginal sonographic measurement of cervical length to predict preterm birth in asymptomatic women at increased risk: a systematic review. Ultrasound Obstet Gynecol 2008;31:579-87.

8. Owen J. Evaluation of the cervix by ultrasound for the prediction of preterm birth. Clin Perinatol 2003;30:735-55.

9. lams JD, Goldenberg RL, Meis PJ, Mercer BM, Moawad $A$, Das $A$, et al. The length of the cervix and the risk of spontaneous premature delivery. National Institute of Child Health and Human Development Maternal Fetal Medicine Unit Network. N Engl J Med 1996;334:56772.

10. Owen J, Yost N, Berghella V, Thom E, Swain M, Dildy GA 3rd, et al. Mid-trimester endovaginal sonography in women at high risk for spontaneous preterm birth. JAMA 2001;286:1340-8.

11. Podobnik M, Bulic M, Smiljanic N, Bistricki J. Ultrasonography in the detection of cervical incompetency. J Clin Ultrasound 1988;16:383-91.

12. Barber MA, Medina M, Cabrera F, Romero A, Valle L, Garcia-Hernandez JA. Cervical length vs VOCAL cervical volume for predicting pre-term delivery in asymptomatic women at 20-22 weeks' pregnancy. J Obstet Gynaecol 2012;32:648-51.

13. Jo YS, Jang DG, Kim N, Kim SJ, Lee G. Comparison of cervical parameters by three-dimensional ultrasound according to parity and previous delivery mode. Int J Med 


\section{Obstetrics \& Gynecology Science}

Ji Youn Kim, et al. 3D volumetric uterine cervix histogram prediction of delivery

Sci 2011;8:673-8.

14. Dilek TU, Gurbuz A, Yazici G, Arslan M, Gulhan S, Pata O, et al. Comparison of cervical volume and cervical length to predict preterm delivery by transvaginal ultrasound. Am J Perinatol 2006;23:167-72.

15. Hoesli IM, Surbek DV, Tercanli S, Holzgreve W. Three dimensional volume measurement of the cervix during pregnancy compared to conventional 2D-sonography. Int J Gynaecol Obstet 1999;64:115-9.

16. Park IY, Kwon JY, Kwon JY, Hong SC, Choi HM, Kwon HS, et al. Usefulness of cervical volume by three-dimensional ultrasound in identifying the risk for preterm birth. Ultrasound Med Biol 2011;37:1039-45.

17. Rozenberg P, Rafii A, Senat MV, Dujardin A, Rapon J, Ville $Y$. Predictive value of two-dimensional and threedimensional multiplanar ultrasound evaluation of the cervix in preterm labor. J Matern Fetal Neonatal Med 2003;13:237-41.

18. Furtado MR, Pires CR, Araujo Junior E, De Souza E, Nardozza LM, Moron AF. Transvaginal grey scale histogram of the cervix at 20-25 weeks of pregnancy. Aust N Z J Obstet Gynaecol 2010;50:444-9.

19. Kuwata T, Matsubara S, Taniguchi N, Ohkuchi A, Ohkusa T, Suzuki M. A novel method for evaluating uterine cervical consistency using vaginal ultrasound gray-level histogram. J Perinat Med 2010;38:491-4.

20. Tekesin I, Hellmeyer L, Heller G, Romer A, Kuhnert M, Schmidt $\mathrm{S}$. Evaluation of quantitative ultrasound tissue characterization of the cervix and cervical length in the prediction of premature delivery for patients with spontaneous preterm labor. Am J Obstet Gynecol 2003; 189:532-9.

21. Tekesin I, Wallwiener D, Schmidt S. The value of quantitative ultrasound tissue characterization of the cervix and rapid fetal fibronectin in predicting preterm delivery. J Perinat Med 2005;33:383-91.

22. Jorn H, Kalf K, Schwann R, Rath W. Grey-scale texture analysis of the cervix in pregnancy. Ultraschall Med 2006;27:347-54.

23. Rovas L, Sladkevicius P, Strobel E, Valentin L. Reference data representative of normal findings at two-dimensional and three-dimensional gray-scale ultrasound examination of the cervix from 17 to 41 weeks' gestation. Ultrasound Obstet Gynecol 2006;27:392-402.

24. Rovas L, Sladkevicius P, Strobel E, Valentin L. Intraobserver and interobserver reproducibility of three-dimensional gray-scale and power Doppler ultrasound examinations of the cervix in pregnant women. Ultrasound Obstet Gynecol 2005;26:132-7.

25. Yilmaz NC, Yigiter AB, Kavak ZN, Durukan B, Gokaslan H. Longitudinal examination of cervical volume and vascularization changes during the antepartum and postpartum period using three-dimensional and power Doppler ultrasound. J Perinat Med 2010;38:461-5.

26. Rath W, Osmers R, Stuhlsatz HW, Adelmann-Grill BC. Biochemical principles of cervix ripening and dilatation. Z Geburtshilfe Perinatol 1994;198:186-95. 